\title{
A Non Intrusive Human Fatigue Monitoring System
}

\author{
Muhammad Jafar Ali, Suvra Sarkar, GNVA Pavan Kumar, and John-John Cabibihan
}

\begin{abstract}
An algorithm for the real time detection of human fatigue has been developed by using multiple fatigue parameters. In addition to existing metrics such as Blink rate and the PERCLOS, a third metric, the variation of head position and the tilt has been used in generating a cumulative measure of fatigue with non intrusive techniques of monitoring. The initial results obtained out of the simulation studies have been discussed in this paper with a stress on fatigue monitoring using variation in head position. The system was found to be sufficiently accurate and highly robust in prediction of fatigue under test situations for fatigue monitoring.
\end{abstract}

Index Terms-Fatigue monitoring, PERCLOS, algorithm.

\section{INTRODUCTION}

Fatigue can be broadly defined as a feeling of weariness, tiredness or lack of energy. Fatigue is a common complaint but, in medical terms, it may be a possible symptom or cause of other conditions than as a condition itself. While fatigue may be common to all, but studies show that young people, shift workers and people with sleep disorders such as sleep apnoea and insomnia are more prone to such problems [1]. Studies conducted on workplace fatigue have shown to have had severe impact in the long run. The fatigue study conducted by Ricci et al. [2] was the first to examine the relationship between fatigue and health-related lost productive work time (LPT) in U.S. workers. Overall, 9.2\% of U.S. workers with fatigue reported LPT specifically due to fatigue in the previous two weeks. Such workers lost an average of 4.1 productive work hours per week, most of which was reflected in reduced performance at work rather than absence from work. The study concluded that fatigued employees cost U.S. employers $\$ 136.4$ billion per year in health-related LPT-\$101 billion more than workers without fatigue. The impact of long hours of work and fatigue on health related problems and injuries ranging from minor workplace incidents to severe accidents has been studied in detail by Allen et al [3]. Fatigue induced accidents are also not new or never heard of. In recent times there have been an increasing number of incidents being reported with severe casualties along with substantial damage of property. The US Department of Transportation's investigations into fatigue during as early as the 1990s revealed that the extent of fatigue-related fatal accidents is estimated to be around $30 \%$. Research shows that driver fatigue is a significant factor in approximately $20 \%$ of commercial road transport crashes and over $50 \%$ of long haul drivers have fallen asleep at the wheel.

Manuscript received April 6, 2012; revised May 20, 2012.

Muhammad Jafar Ali is with the Department of Mechanical Engineering, National University of Singapore, Lower Kent Ridge Road, Singapore 119260 (e-mail: mjafarmct@gmail.com, Tel: 85067178).

Suvra Sarkar, GNVA Pavan Kumar, and John-John Cabibihan are with the Department of Electrical and Computer Engineering, National University of Singapore, Lower Kent Ridge Road, Singapore 119260.
Much effort has been dedicated towards development of active safety systems for real time fatigue monitoring which can be classified into certain broadly defined categories [4] such as performance based or through measurement of ocular physiology [5], mathematical models of alertness dynamics joined with ambulatory technologies and in-vehicle real time fatigue monitoring based on biobehavioral dimension(s) of an operator, such as features of the eyes, face, head, heart, brain activity, reaction time, etc., during driving [6-8] based on one form or another of the various techniques for fatigue monitoring and correlation. Amongst all the techniques reported so far, perhaps the best indicator would be based on decisions generated on the basis of brain waves, heart rates and pulse rates, which require strong subject coordination with the online tracking procedure such as some form of body appendage or sensor, is costly to implement and in many situations is not feasible due to the intrusion and annoyance caused to the subject in real world scenarios. The results so far based on techniques that monitor eyelid movement and eye gaze with a head-mounted eye tracker or special contact lens have been quite successful. Also reports on monitoring head movement [9] with a head-mount device are also encouraging. The efforts so far are mostly directed towards detecting driver fatigue, but they could be extended in other similar scenarios as well. Ishii et al. [13] introduced a system for characterizing a driver's mental state from his facial expression. A fatigue monitoring system based on line of sight of gaze was reported by Satio et al. [10] Eyelid movements have also been studied by Bovere et al[12] and has been successfully used in fatigue monitoring. The results obtained so far have been quite promising in detecting driver fatigue. Ueno et al. [11] proposed a system for drowsiness detection by recognizing whether a driver's eyes are open or closed and, if open, computing the degree of eye openness. Although, most of these methods based on non-intrusive tracking of the subject are quite promising, there has been substantially less work on fatigue monitoring based on head position and movement. Though there have been some studies on measuring the nodding frequency (NodFreq), very few have been reported and not have been as successful. And, to our knowledge there are very few reports on real time fatigue monitoring based on head tilt, position and variance. We also propose to combine the fatigue prediction using head data with other standard fatigue metrics such as blink rate and percentage closure of the eye (PERCLOS) in the development of a fatigue monitoring system based on simultaneous tracking of multiple parameters which could be an indication of the level of fatigue in an individual. The system proposed here will non-intrusively track, and in real time monitor several visual behaviors that typically characterize a person's level of alertness, and the system could be used in fatigue monitoring in the context of 
preventing vehicular accidents as well as increasing productivity in employees by providing an indication of their level of fatigue. The composite fatigue index would robustly, accurately, and consistently characterize one's vigilance level.

\section{FAtigue Monitoring SYSTEM AND PREDiction ALGORITHM}

The fatigue monitoring algorithm developed by us uses the Seeing Machines Facelab 5 eye and facial tracking solution [14] to track the subject in real time. The setup comprises a stereo head that has two high precision cameras to continuously grab image frames of the subject being tracked. The head or position tracking system particularly uses two coordinate reference frames, the world or the stereo head reference frame and the head reference frame. The stereo head and the world reference frame include the sensor while the subject reference frame includes the subject head reference frame. The Head-Pose is measured relative to the World Coordinate Frame. The sensor determines the Head-Pose by transforming the Head Coordinate Frame into the World Coordinate Frame through the relation $\mathrm{X}_{\mathrm{w}}=\mathrm{RX}_{\mathrm{H}}$ $+\mathrm{T}$. The Head-Pose is actually the combination of the rotation matrix $R$ and the translation vector $T$. The rotation matrix $\mathrm{R}$ is outputted by faceLAB in the form of three Euler angles and the translation vector $\mathrm{T}$ is outputted in the form of three vector elements. These coordinates so obtained can be used to perform calculations and predictions using an algorithm. The facelab system basically employs an edge detection algorithm to track the subject features and position.

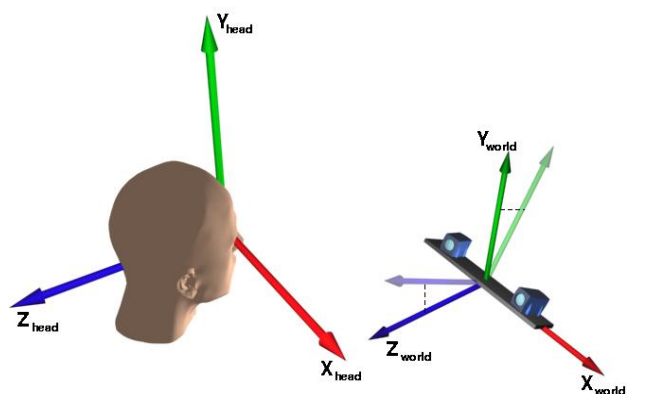

Fig. 2. The head coordinate reference frame of the tracking setup

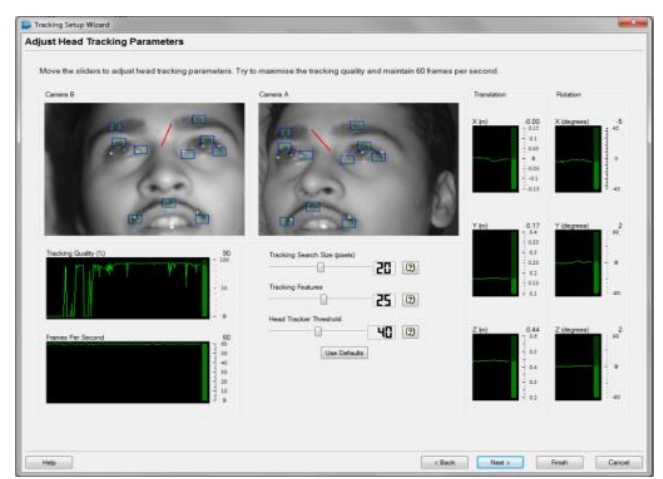

Fig. 3. Calibration of subject features for tracking

In our setup for tracking we have used the blink rate and the PERCLOS value in combination with the head pose and variation to predict levels of fatigue in an individual. The blink measurement in the sensor used here is a binary signal (true or false), which reports the occurrence of blink events. They are defined as a rapid eye closure followed quickly by a rapid eye opening. The binary signals can then be used for analysis in further stages. In addition to the blink value (true or false), we have also used the PERCLOS value to increase the tracking confidence and the probability of getting a correct prediction. The PERCLOS fatigue measure is regarded as the most reliable single visible indicator for fatigue. It is based on the eye closure of the subject, in particular the percentage of extended periods with practically closed eyes in a time window of fixed size. The method specifically discounts "regular" eye blinks which must be excluded from the data. The intricate details of the working of the facelab are beyond the scope of this paper and can be found in the reference [14]. All the data obtained through the sensor are transmitted via the TCP/IP protocol to a slave machine that runs an intelligent prediction algorithm to infer upon the level of fatigue of the subject being tracked. The algorithm has been developed by us using the CoreData Application Programming Interface [14] to access the class members in the data packets sent via the network, which point to the various parameters such as the head pose in 3 coordinates, the blink value and the PERCLOS. The prediction algorithm will continuously receive the frames containing the composite data transmitted by the sensor. Methods were designated to compute the variance in the data, obtain the mean value over a period of time and as well, perform comparisons with predefined threshold values for each metric used in fatigue monitoring. Depending upon the judgment obtained from the prediction of the fatigue level a signal is transmitted via the serial port to a microcontroller based triggering circuit.

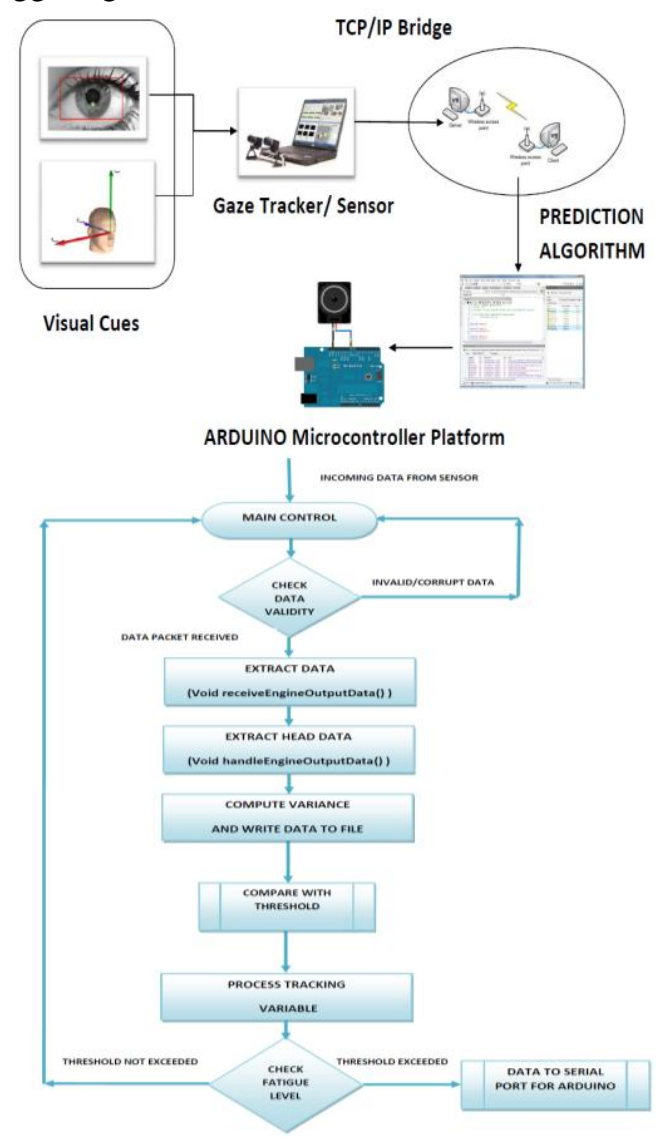

Fig. 4. Block diagram of the setup used for fatigue monitoring and the flow chart of the concept implemented

The performance of the system under composite decisions 
has not been dealt with in this work. In order to test the viability of the system, real life situations were simulated on the laboratory scale using a subject who was asked to perform activities similar to ones reported in fatigued individuals based on the literature available. Initially the system was configured by setting a model for the subject on the facelab machine, by accurately choosing coordinate points on the subject face.

In order to have a standard or threshold of the head position and movement we first obtained 3000 successive data frames for an extended period over which the variation was calculated. During the course of data recording the subject head movement was purposely kept to a bare minimum which would be normal to a healthy human being. A reading of $10 \mathrm{nod} / \mathrm{min}$ is usually taken as an indication of sleepiness but this threshold level still requires further verification. It is also believed that the head tilt of an individual is a monitor of the fatigue and that at the onset of fatigue the head of an individual tilts by a certain degree. In order to incorporate the sensing of complete loss of alertness by an individual we also monitor the mean head position over time. The head tilt tracking was performed on three typical positions of sleep while on the sleeping posture, a downward tilt and a left and a right tilt. This data is compared to a threshold value for the person's head tilt which is also measured while taking the standard data. In addition to this, as stated earlier, the successive blink frequency and as well as the PERCLOS value are also simultaneously tracked. Combining all this data, the proposed algorithm makes a decision on the level of fatigue and generates an alarm to the subject.

\section{RESULTS AND DISCUSSIONS}

The initial set of tests performed on simulated fatigue scenarios produced substantially good results with a high degree of accuracy. The plots for the position obtained under non-fatigued conditions show a very minimal change in the head position over time. Though, we should mention here that a tolerance must be kept in order to take into consideration the sudden movements of the head which is very normal to an individual. The variance of the data sets so obtained has been shown below in the Table I. An average value obtained out this data was used for setting a threshold, beyond which any continuous head movement over time would be a reasonably accurate indicator of the onset of fatigue. A typical data set obtained under simulated fatigue conditions has been shown in the Table II. Also, in order to test the real time efficiency of this system, a lower set of frames was chosen and the subject was made to perform a predefined set of movements generally characteristic of a fatigued individual.

The system could indicate the exceeding of the threshold limit via sound signals generated through the Arduino interface at appropriate situations, and this was consistently tested successful (Video of tracking not shown here). In order to account for the changes in variance due to normal stretching movement and sudden movements, and hence to prevent a false alarm, an indicator was used in the algorithm which kept a record of the increase in the variance of the 3000 frame sets received every time.
Head Position (x Co-Ordinate)
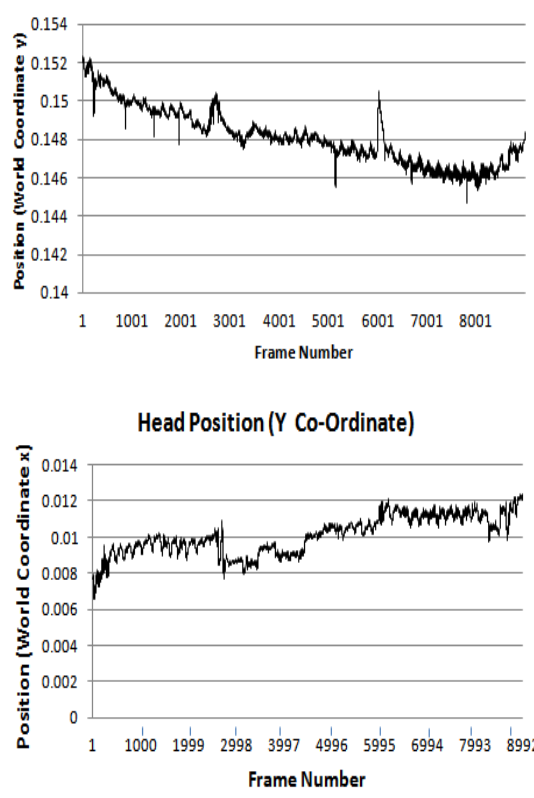

Head Position (2 Co-Ordinate)

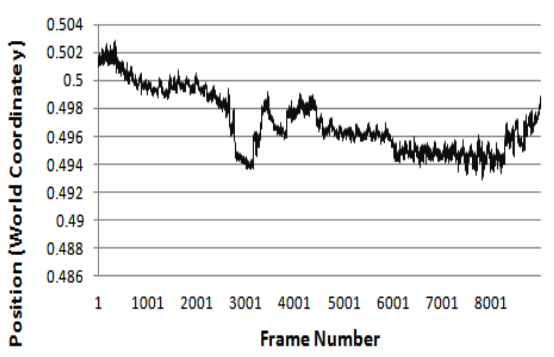

Fig. 5. Head position data under non-fatigued conditions

TABLE I AND II: VARIANCE IN HEAD DATA UNDER SIMULATED NON FATIGUED AND FATIGUED CONDITIONS FOR 3000 FRAME SETS RESPECTIVELY

\begin{tabular}{|l|l|l|}
\hline X (Variance) & Y (Variance) & Z(Variance) \\
\hline $4.79527 \mathrm{e}-007$ & $8.22058 \mathrm{e}-006$ & $8.32988 \mathrm{e}-005$ \\
\hline $4.23483 \mathrm{e}-007$ & $8.05874 \mathrm{e}-006$ & $8.21132 \mathrm{e}-005$ \\
\hline $4.91204 \mathrm{e}-007$ & $8.04006 \mathrm{e}-006$ & $8.20017 \mathrm{e}-005$ \\
\hline $4.03098 \mathrm{e}-007$ & $7.92229 \mathrm{e}-006$ & $8.05405 \mathrm{e}-005$ \\
\hline
\end{tabular}

\begin{tabular}{|c|c|c|}
\hline (Variance) & Y (Variance) & $\mathbf{Z}$ (Variance) \\
\hline 0.000801321 & $4.72956 \mathrm{e}-005$ & 0.000112562 \\
\hline 0.000501846 & $7.68592 \mathrm{e}-005$ & 0.000330436 \\
\hline 0.000217722 & $6.55998 \mathrm{e}-005$ & 0.000288281 \\
\hline
\end{tabular}

In addition, in order to prevent a false alarm from being generated the indicator threshold was kept sufficiently high by taking an average of the variance values obtained under standard conditions so that only in situations where the subject's head position has changed continuously above the variance, the alarm was raised, signalling the onset of fatigue. In the data sets shown in the head position variance tests, we observe that there is comparatively lesser variation in the head position along the $y$-axis. Here we may point out that the movement of the head is typical of an individual and that it may vary from one person to the other under fatigue scenarios. As such the variance in movement along all the 
three coordinates is continuously monitored to obtain a decision. The tracking of the mean head position/tilt was also substantially accurate over long durations and the system did respond fairly accurately to the simulation setups. The tilt seems to be a reasonably good metric, it is again felt that the degree of head tilt will vary from one individual to another and the coordinate based decision might be prone to error. Hence this particular metric would require more in-depth analysis and the use of real subjects for validity.

In addition to the tracking of the head position and the variation in head position, the blink as well as the PERCLOS was obtained and was tested to monitor the fatigue levels. The simulated data set has been shown below; wherein the blink rate is fairly high during the initial stages indicating a reasonably high level of alertness while a substantial drop can be observed in the later stage which is an indication of the onset of fatigue. The PERCLOS or the percentage closure of the eyes in a specified time window was also seen to rise significantly under the simulation studies conducted where the subject imitated the gradual onset of fatigue with time which was reflected in increasing closure times of the eye in a time interval. The plot of the PERCLOS data so obtained has also been shown below in the following pages. The eye data obtained by us under simulated fatigue scenarios is quite similar to those obtained by $\mathrm{Ji}$ et al. [15]. It is quite established that the eye data is a highly accurate indicator of the fatigue level in an individual. Simultaneous detection of

all these parameters is used to accurately judge the onset of fatigue in an individual. It has been depicted through the simulations that the tracking system is fairly robust in the prediction and that a combination all these fatigue parameters produced significantly accurate signal of the level of alertness of the individual.

TABLE III: RIGHT TILT IN HEAD OVER TIME.

\begin{tabular}{|c|c|c|}
\hline X (Mean) & Y (Mean) & Z(Mean) \\
\hline 0.0166056 & 0.173882 & 0.517850 \\
\hline 0.0164633 & 0.173709 & 0.519029 \\
\hline 0.0167570 & 0.174270 & 0.520014 \\
\hline
\end{tabular}

TABLE IV: LEFTWARD TILT IN HEAD OVER TIME.

\begin{tabular}{|c|c|c|}
\hline X (Mean) & Y (Mean) & Z(Mean) \\
\hline-0.0315605 & 0.184011 & 0.513198 \\
\hline-0.0308553 & 0.182989 & 0.512089 \\
\hline-0.0312637 & 0.183471 & 0.514978 \\
\hline
\end{tabular}

TABLE V: DOWNWARD TILT IN HEAD OVER TIME.

\begin{tabular}{|c|c|c|}
\hline X (Mean) & Y (Mean) & Z(Mean) \\
\hline-0.00822589 & 0.162898 & 0.441961 \\
\hline-0.00922814 & 0.162759 & 0.442920 \\
\hline-0.00616837 & 0.166079 & 0.443674 \\
\hline
\end{tabular}
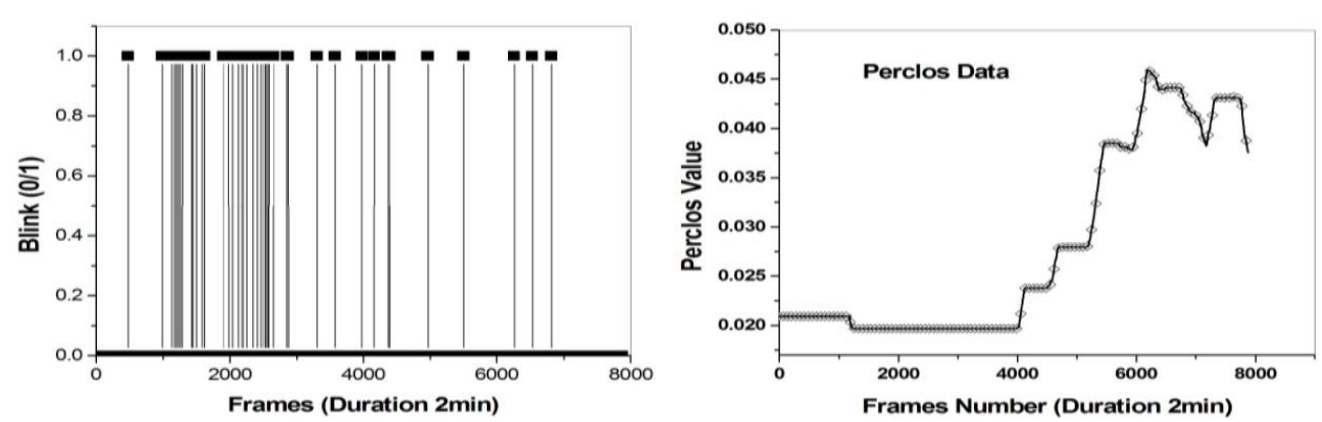

Fig. 6. Blink and PERCLOS data for a period of two minutes with 3000 frame sets under simulated situations of onset of fatigue.

\section{CONCLUSION}

A real time non-intrusive fatigue monitoring system has been proposed based on simultaneous tracking of multiple fatigue metrics. The results obtained on real time fatigue monitoring based on variation in head position was found to be substantially accurate under simulated situations. The decision algorithm was also found to be quite accurate but would need more rigorous studies, which is part of a future work. The cumulative decision system proposed here based on all the fatigue parameters could be of immense importance in real life situations in fatigue monitoring and mitigation.

\section{ACKNOWLEDGEMENT}

The authors wish to thank Prof. John-John Cabibihan for agreeing to work on this project and for his help, encouragement and enthusiasm throughout the experiments. The authors are thankful to National University of Singapore, for providing all the facilities and resources during the course of this experiment.

\section{REFERENCES}

[1] Transport Accident Commission, Fatigue Case Study. http://www.tac.vic.gov.au

[2] Ricci, Chee, Lorandeau, and Berger, "Fatigue in the U.S. Workforce: Prevalence and Implications for Lost Productive Work Time," Journal of Occupational and Environmental Medicine, vol. 49, no. 1, Jan. 2007.

[3] Allen, Slavin, and Bunn, "Do Long Workhours Impact Health, Safety, and Productivity at a Heavy Manufacturer," Journal of Occupational and Environmental Medicine, vol. 49, no. 2, Feb. 2007.

[4] L. Hartley, T. Horberry, N. Mabbott, and G. Krueger, "Review of Fatigue Detection and Prediction Technologies," Melbourne, Australia: National Road Transport Commission, 2000.

[5] D. Dinges and M. Mallis, "Managing fatigue by drowsiness detection: Can technological promises be realized? In managing fatigue in transportation," in Managing Fatigue in Transportation: Selected Papers from the 3rd Fatigue in Transportation Conference, remantle, Western Australia, L. R. Hartley, Ed. Oxford, U.K.: Elsevier, 1998, pp. 209-229.

[6] E. Grandjean, Fitting the Task to the Man, 4th ed. London, U. K. : Taylor and Francis, 1988.

[7] D. Cleveland, "Unobtrusive eyelid closure and visual point of regard measurement system," presented at the Proc. Ocular Measures Driver Alertness Tech. Confe., Herndon, VA, Apr. 26-27, 1999. 
[8] R. J. E Carroll, "Ocular measures of driver alertness technical conference proceedings," Federal Highway Administration, Office of Motor Carrier and Highway Safety, Washington, DC, FHWA Tech. ep. FHWA-MC-99-136, 1999.

[9] Appl. Sci. Lab., "PERCLOS and eyetracking: Challenge and opportunity," Tech. Rep., Appl. Sci. Lab., Bedford, MA, 1999.

[10] H. Saito, T. Ishiwaka, M. Sakata, and S. Okabayashi, "Applications of driver's line of sight to automobiles-what can driver's eye tell," in Proc. Vehicle Navigation Information Systems Conf., Yokohama, Japan, Aug 1994, pp. 21-26.

[11] H. Ueno, M. Kaneda, and M. Tsukino, "Development of drowsiness detection system," in Proc. Vehicle Navigation Information Systems Conf., Yokohama, Japan, Aug. 1994, pp. 15-20.
[12] S. Boverie, J. M. Leqellec, and A. Hirl, "Intelligent systems for video monitoring of vehicle cockpit," in Proc. Int. Congr. Expo. ITS: Advanced Controls Vehicle Navigation Systems, 1998, pp. 1-5.

[13] T. Ishii, M. Hirose, and H. Iwata, "Automatic recognition of driver's facial expression by image analysis," J. Soc. Automotive Eng. Japan, vol. 41, no. 12, pp. 1398-1403, 1987

[14] Facelab 5 is a product of Seeing Machines, Australia. www.seeingmachines.com

[15] Q. Ji, Z. W. Zhu, and P. Lan, "Real-Time Nonintrusive Monitoring And Prediction of Driver Fatigue," IEEE Transactions on Vehicular Technology, vol. 53, no. 4, July 2004. 